\title{
The effects of arm length, size of angle, and spatial separation on Morinaga's illusion
}

\author{
O. L. TENNANT and A. E. WILSON \\ University of New Brunswick, Saint John, New Brunswick, Canada
}

\begin{abstract}
The effects of arm length, spatial separation between angles, size of angle, and practice on the degree of misalignment in a modified version of Morinaga's illusion were assessed. Size of angle was manipulated between subjects ( $n=15$ /group), whereas arm length, spatial separation, and practice were varied within subjects. Results showed: (1) that as the length of the arm increased, the degree of illusion decreased; (2) that as spatial separation increased, the degree of illusion increased; and (3) an interaction between size of angle and practice. The results were related to Day et al.'s (1982) assimilation theory of Morinaga's illusion.
\end{abstract}

Morinaga's illusion refers to an apparent misalignment of objectively aligned apices of angles. Figure 1, which depicts the illusion, reveals that the apices of angles appear displaced in the direction in which the angles point. Therefore, to subjectively align the apices A, B, C, and $D$, either angles $A$ and $C$ should be positioned toward the left or angles B and D should be positioned to the right.

The illusion initially attracted interest because of a perceived paradox between apparent alignment and subjective distance (Robinson, 1972). In Figure 1, the distance between $A$ and $A_{1}$ appears greater than the distance between $B$ and $B_{1}$, although both distances are objectively equal. This alteration in perceived distance is the wellknown Müller-Lyer illusion. Since the distance between the apices of the inward fins appears shorter than the distance between the outward fins, one would logically assume that the apices of the inward fins would appear displaced inward relative to the apices of the outward fins. This is not the case, since, as noted above, the apices of inward fins appear displaced in the opposite direction. Recently, Restle (1976) demonstrated that Morinaga's configuration does not involve a true paradox. He found that the illusion of misalignment occurs independently of the illusion of length. As shown in Figure 1b, the apparent misalignment is present with one column of components.

Day, Bellamy, and Norman (1982) have recently explained the apparent misalignment on the basis of assimilation. In brief, they argue that the alignment of the apex of an angle assimilates to the region of overlap between that angle and the adjacent element of a neighboring angle. Consider, for example, the three-angled variant of Morinaga's illusion displayed in Figure 2. In A, the

\footnotetext{
This article is based on an honors thesis submitted to the University of New Brunswick. The research was supported by the Natural Sciences and Engineering Research Council of Canada by a grant (A8323) to the senior author. Portions of this paper were presented at CPA (Halifax, Nova Scotia, 1985). Requests for reprints should be sent to A. E. Wilson, Division of Social Sciences, University of New Brunswick, Saint John, New Brunswick, Canada E2L 4L5.
}

regions of overlap of the center apex, $X$, are MYX and $X N Z$. In $B$, the regions of overlap of apices $Y$ and $Z$ are $\mathrm{XOY}$ and $\mathrm{XPZ}$, respectively. In each case, the direction of misalignment is in the direction of overlap, namely, $\mathrm{X}$ appears positioned too far to the left to appear aligned with $\mathrm{Y}$ and $\mathrm{Z}$, and $\mathrm{Y}$ and $\mathrm{Z}$ appear to line up to the left of $\mathrm{X}$.

The principle of assimilation used by Day et al. (1982) is the same one invoked to explain other illusions, such as the Müller-Lyer and the Delboeuf. For the Müller-Lyer illusion, it is argued that the distance between the apices of the angles assimilates to the distance between the fins, and for the Delboeuf illusion, it is argued that the size of the inner circle assimilates to the size of the larger circle.

Day et al. found an increased degree of misalignment in Morinaga's illusion as the size of the components decreased and as the space between the components increased. They noted that the regions of overlap between the apex of the center angle and its adjacent components

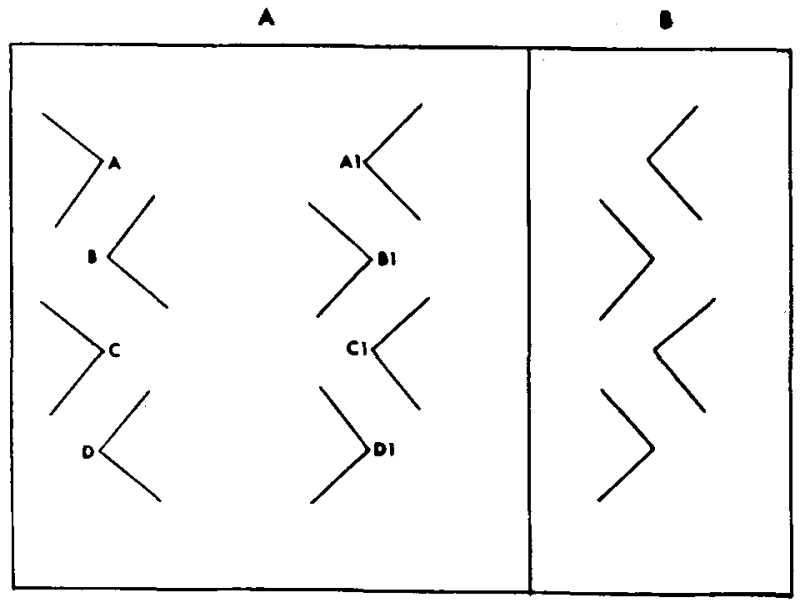

Figure 1. (A) In Morinaga's illusion, the apices of the angles do not appear aligned. (B) The illusion is present with one column of components. 
A

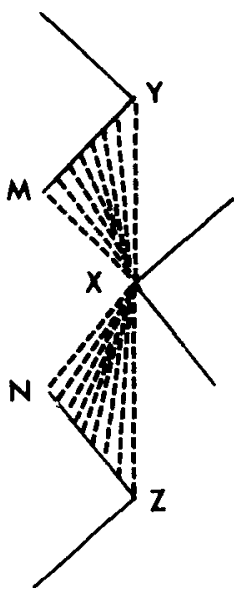

B

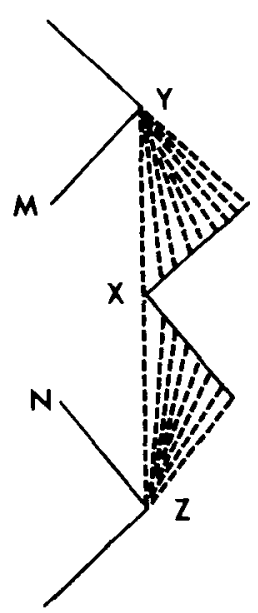

Figure 2. The shaded areas denote the overlap of the apices of angles with neighboring angles.

decreased in both conditions, and suggested that the degree of assimilation and the magnitude of illusion was greatest when the overlap was slight. ${ }^{1}$ They maintained that these results were consistent with past findings obtained with illusions such as the Müller-Lyer and the Delboeuf, in which Day (1977) reported the degree of illusion to be greatest when the size of the whole figure was only slightly greater than the component of the figure to be judged. According to Day et al. (1982), the illusion should be larger when the overlap between the apex of an angle (the target feature) and its adjacent component is slight than when it is large.

A question can be raised in regard to how close the correspondence is between Day et al.'s findings with Morinaga's illusion and results obtained with illusions that have typically been explained on the basis of assimilation. Generally, it is reported that as the size of the context increases, the degree of illusion initially increases and then decreases (Brigell, Uhlarik, \& Goldhorn, 1977). Thus, if there is a close correspondence between Morinaga's and other illusions, one might predict that if size of overlap increased, the degree of misalignment would initially increase and then decrease. If the predicted relationship were found, it would provide support for the idea that the illusion could be explained on the basis of assimilation.

The most obvious way to manipulate degree of overlap is to vary the length of the arms of the angles. When Day et al. varied arm length, they found an inverse relationship between degree of misalignment and length of arm. However, they used only two values of arm length, and it is conceivable that their results reflected their choice of a limited selection of extents and that, with a greater selection of arm lengths, the nonmonotonic function obtained with other illusions of assimilation might be found. The primary purpose of this study, therefore, was to verify the functional relationship between degree of illusion and arm length.

This experiment also varied the distance between angles and the size of the acute angle. Day et al. found that Morinaga's illusion increased as the distance between angles increased, and Restle (1976) found that the largest illusion occurred with an angle of $30^{\circ}$. A secondary purpose of this study was to verify the effects of the size of angle and spatial separation on the degree of illusion.

\section{METHOD}

\section{Subjects}

Forty-five introductory psychology students, who reported normal or corrected-to-normal vision, participated in this experiment. Course credit was awarded to participants.

\section{Materials}

Stimulus figures were drawn in black ink on separate $21.1 \times$ $27.6 \mathrm{~cm}$ sheets of white paper and then duplicated by an offsetting procedure. As shown in Figure 3, the experimental figures consisted of two angles, with the arms of the angles pointing in opposite directions. Among displays, the size of the angle, the length of the arms of the angles, and the distance between the apices of the angles varied. The sizes of the angles were $15^{\circ}, 30^{\circ}$, and $45^{\circ}$. The lengths of the arms were $5,10,20,35,55$, and $80 \mathrm{~mm}$. The distances between the apices of the angles were 35 and $75 \mathrm{~mm}$. The apices of the angles were centered $10.6 \mathrm{~cm}$ from each edge of the sheet.

To control for response bias, two control displays were constructed. They consisted of two dots located in a position corresponding to the location of the apices of angles in the experimental displays. For one control display, the dots had a $35-\mathrm{mm}$ separation; in the other, there was a $75-\mathrm{mm}$ separation.

At the bottom of each display, a horizontal response line was drawn. The line was positioned $25 \mathrm{~mm}$ from the bottom of the sheet and extended the entire width of the page. The distance between the line and the bottom apex of the experimental figures or the lower dot of the control figures was $100 \mathrm{~mm}$.

During testing, the figures were centered on a $45.5 \times 159.5 \mathrm{~cm}$ white target holder. The face of the holder was tilted $20^{\circ}$ away from
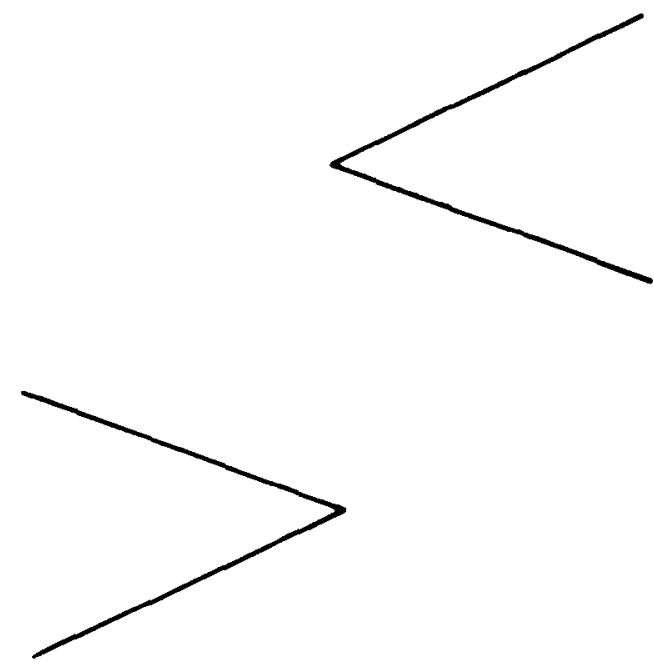

Figure 3. An example of the stimulus figures used in the experiment. 
the subject. A chinrest was located directly in front of the target holder so that the distance between the eyes of the subject and the target was approximately $45 \mathrm{~cm}$.

\section{Experimental Design}

A $3 \times 6 \times 2 \times 2$ mixed factorial design was used. Angle size varied between subjects ( $n=15$ per group), and arm length, spatial separation, and practice varied within subjects.

\section{Procedure}

After signing a consent form, the subject was informed that his or her task was to place a dot on the response line such that it appeared aligned either with the apices of the angles of the experimental figures or with the centers of the dots of the control figures. Sample figures were used to demonstrate the task. The experimenter emphasized that hand movements were to be used only to make the response and not as an aid in judging alignment.

Each subject was tested on two consecutive sequences of 14 displays. Each sequence of displays consisted of a randomized order of the two control figures and the 12 experimental displays formed by combining the two levels of spatial separation with the six levels of arm length.

During testing, the subject placed his or her chin on the chinrest to view the stimulus figures. The rate of presentation of the figures was subject paced such that the speed of presentation depended upon the amount of time taken by each subject to make the required judgment.

\section{RESULTS}

For each display, the degree of angular distortion (Pressey \& Sweeney, 1972) of the response from objective alignment with either the apices of angles in the experimental figures or the center of the dots in the control figures was measured with a protractor. Responses consistent with Morinaga's illusion, counterclockwise devi-

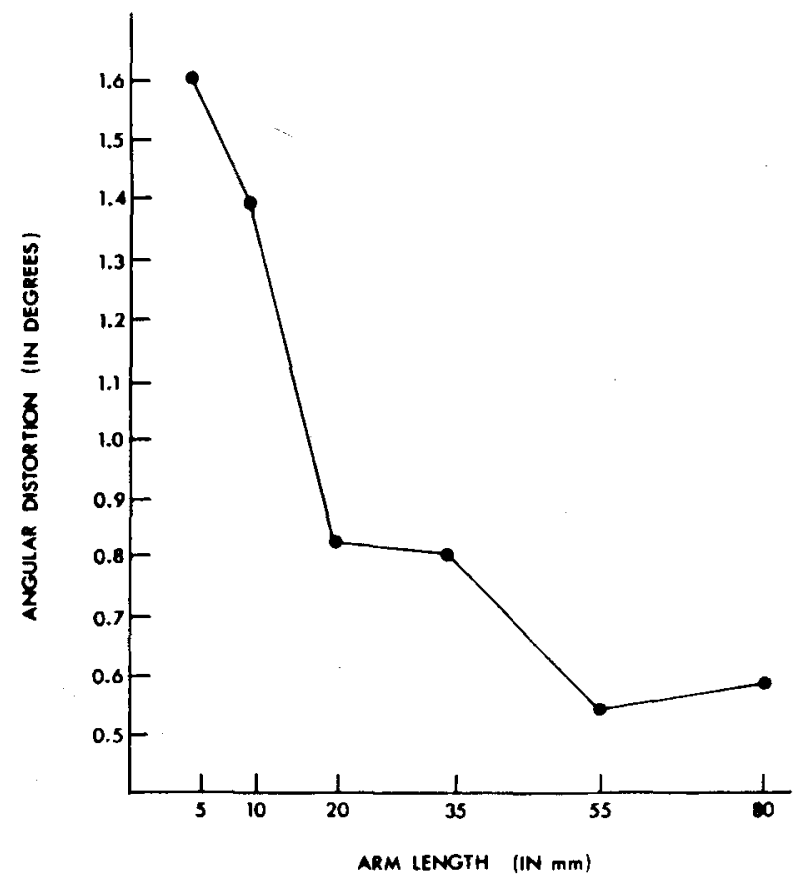

Figure 4. Mean degree of illusion as a function of arm length.

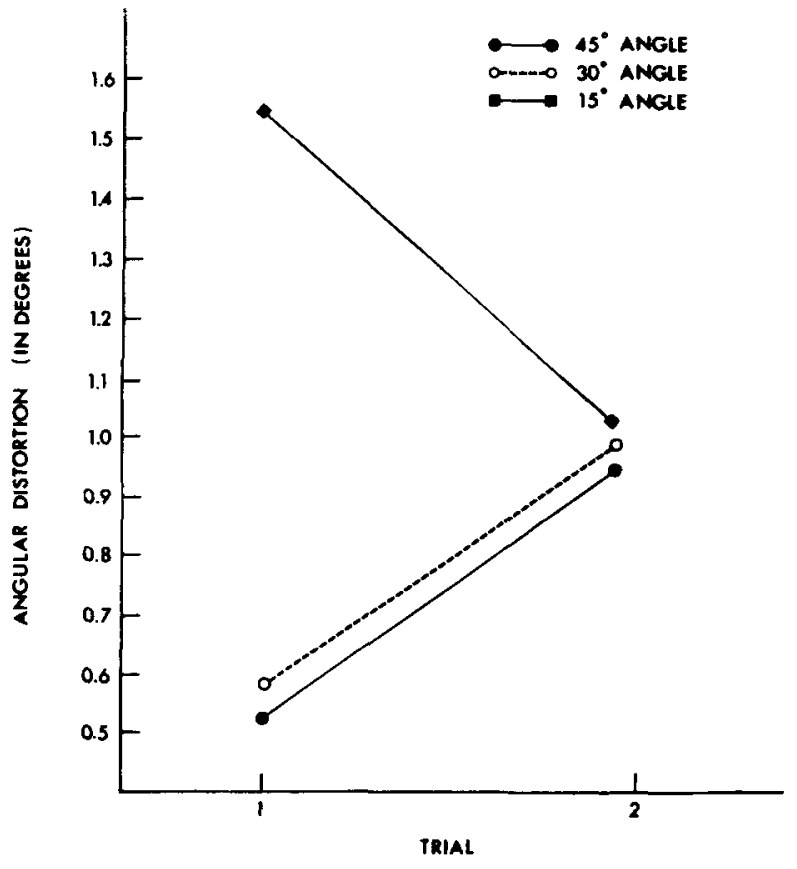

Figure 5. Mean degree of illusion as a joint function of size of angle and practice.

ation from veridicality, were scored as positive; responses inconsistent with the illusion, clockwise deviations, were scored as negative. The degree of illusion for each response was then calculated by subtracting the score of the appropriate control figure from the score of the experimental figure.

The illusion scores were submitted to a $3 \times 2 \times 2 \times 6$ ANOVA. The ANOVA revealed that the main effect of arm length $[F(5,210)=11.93, p<.001]$ and the interaction between size of angle and practice $[\mathrm{F}(2,42)=5.53$, $\mathrm{p}=.017]$ were statistically significant. The ANOVA also indicated that the effect of spatial separation approached statistical significance $[F(1,42)=3.04, p=.088]$. All other main effects and interactions failed to attain statistical significance $(p>.05)$.

Figure 4 depicts the mean degree of illusion as a function of arm length. The figure shows that the magnitude of illusion was inversely related to arm length. When the length increased from 5 to $20 \mathrm{~mm}$, the rate of decline was rapid, but with further increases in arm length, the rate of decline in the degree of illusion decreased.

The interaction between angle size and practice is depicted in Figure 5. Visual inspection of the figure suggests that, on the first sequence of testing, the angle of $15^{\circ}$ induced a larger illusion than did displays with angles of either $30^{\circ}$ or $45^{\circ}$. Further inspection also indicates that the degree of illusion decreased with practice for displays with an angle of $15^{\circ}$ but increased for displays with angles of $30^{\circ}$ or $45^{\circ}$. To examine possible sources of the interaction, five post hoc ANOVAs were initially conducted. Two involved contrasting the $15^{\circ}$ angle group with the $30^{\circ}$ and $45^{\circ}$ angle groups on Trial 1 
Table 1

Summary of Post Hoc Analysis

\begin{tabular}{lcccc}
\hline \multicolumn{1}{c}{ Analysis } & df & F & p \\
\hline $15^{\circ}$ group vs. $30^{\circ}$ group on Trial 1 & 1,28 & 2.95 & .097 \\
$15^{\circ}$ group vs. $45^{\circ}$ group on Trial 1 & 1,28 & 4.14 & .051 \\
$15^{\circ}$ group Trial 1 vs. Trial 2 & 1,14 & 4.14 & .051 \\
$30^{\circ}$ group Trial 1 vs. Trial 2 & 1,14 & 2.94 & .108 \\
$45^{\circ}$ group Trial 1 vs. Trial 2 & 1,14 & 3.96 & .067 \\
$15^{\circ}$ group vs. $30^{\circ}$ group on Trials 1 and 2 & 1,28 & 7.94 & .009 \\
$15^{\circ}$ group vs. $45^{\circ}$ group on Trials 1 and 2 & 1,28 & 7.00 & .013 \\
\hline
\end{tabular}

and three examined the effects of practice on each size of angle. As shown in Table 1, the probability values associated with each analysis approached, but did not obtain conventional levels of, statistical significance ( $p>$ .05 ). Two additional post hoc ANOVAs were then conducted. One contrasted the $15^{\circ}$ group with the $30^{\circ}$ group on Trials 1 and 2 , and the second contrasted the $15^{\circ}$ group with the $45^{\circ}$ group on Trials 1 and 2 . The results of the ANOVAs, shown in Table 1, revealed a statistically significant interaction between group and trials. These analyses reflected the differences in the change in degree of illusion across trials between the $15^{\circ}$ group and the $30^{\circ}$ and $45^{\circ}$ groups.

As shown in Figure 6, the degree of illusion increased with an increase in spatial separation from 35 to $75 \mathrm{~mm}$. Although the effect of spatial separation just failed to reach conventional levels of statistical significance, the increase in illusion with an increase in spatial separation was consistent across all angle sizes and therefore seems to be reliable.

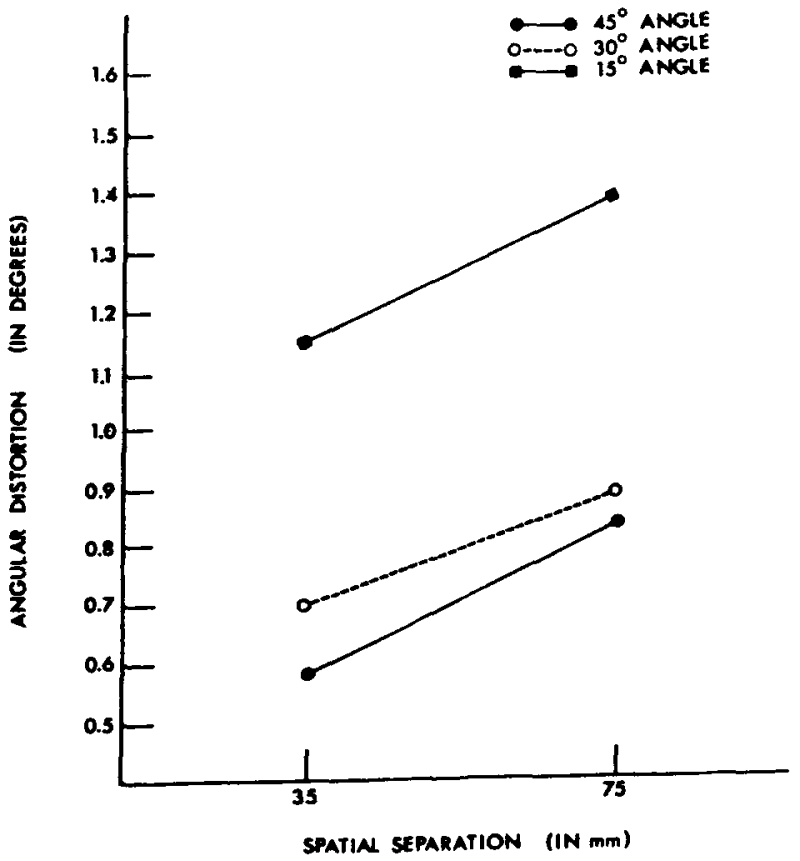

Figure 6. Mean degree of illusion as a joint function of size of angle and spatial separation.

\section{DISCUSSION}

The primary purpose of this experiment was to examine the relationship between the amount of overlap and the degree of misalignment in Morinaga's illusion. Overlap was manipulated by varying arm length. Since Day et al. explained the misalignment on the basis of a principle used to explain such illusions as the Müller-Lyer, it was predicted that the function between arm length and degree of misalignment would mimic the ones obtained with other illusions; that is, it was predicted that if arm length increased, the degree of misalignment would initially increase and then decrease. Contrary to the prediction, the results showed that as arm length increased, the degree of misalignment decreased. If one were to pursue an explanation of Morinaga's illusion based on assimilation, it might be worthwhile to note a major difference between Morinaga's illusion and the ones on which the prediction was based. The major difference is that the former is an illusion of alignment, whereas the latter (e.g., the MüllerLyer and Delboeuf) are ones of extent. It may be that the task required of an observer, that is, judging direction versus judging extent, interacts with the assimilation process to produce different functional relations between degree of illusion and size of context.

This experiment was also designed to verify the results of Day et al., who found that the magnitude of the illusion increased with an increase in spatial separation. Although the results in the current study just failed to attain statistical significance, the illusion did consistently increase with an increase in spatial separation. The dependent variable used in the present study differed from the one used by Day et al. They used linear displacement rather than angular distortion as the dependent variable. It is conceivable that their results reflect the fact that linear displacement would increase simply as a mathematical consequence of increasing distance between the apices of the angles rather than increasing degree of misalignment. To compare the results obtained by Day et al. with those of the current study, their findings were converted to angular distortion. As shown in Figure 7, these results indicate that the degree of misalignment diminishes with short arm lengths $(5 \mathrm{~mm})$, but remains approximately constant with long arm lengths $(20 \mathrm{~mm})$ as spatial separation increases. Since their results differ markedly from those of the present study, one may wish to suspend judgment on the effect of increasing spatial separation on degree of illusion. ${ }^{2}$

Angle size was varied to confirm Restle's (1976) finding that the degree of illusion was inversely related to the size of the angle. Our results indicated that the smallest angle, $15^{\circ}$, induced the largest illusion. Although the effect of angle size did not reach statistical significance, the trend was consistent with Restle's results, which showed that the smallest angle used in his study, $30^{\circ}$, resulted in the largest illusion.

The finding that size of angle interacted with practice is curious. One would think that practice would not 


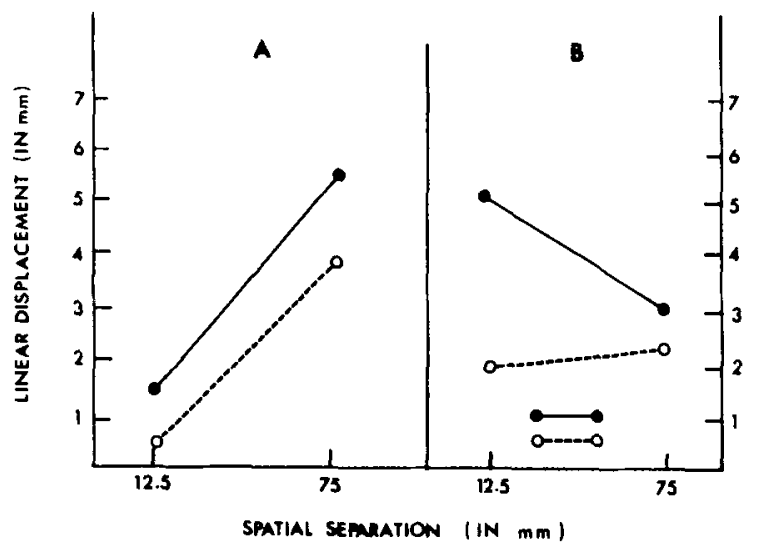

Figure 7. The results of Day, Bellamy, and Norman (1982). In (A), the dependent variable is linear displacement. In (B), the dependent variable is angular distortion.

differentially affect degree of illusion in variants of a display which varied in size of angle. Because post hoc analysis of the main effects associated with the interaction failed to attain conventional levels of statistical significance, the interaction may have resulted from sampling error.

In general, the results are consistent with Day et al.'s explanation, which was based on assimilation of the region of overlap between the apex of an angle and the adjacent component of its neighboring angle. However, their explanation is not precise about what was being assimilated. It is possible that, when an observer judges the alignment of the apices of adjacent angles, one apex assimilates to a position either below or above the adjacent component of the neighboring angle. A second possibility is similar to Pressey's (1971) initial explanation of the Poggendorff illusion. It could be that when a subject attempts to align the apices of angles, he or she projects a series of possible alignments from the apex of one angle to the arm of the overlapping angle and that assimilation occurs among the orientations of the projections. Further research could be directed toward evaluating these two competing explanations.

\section{REFERENCES}

Brigell, M., Uhlarik, J., \& Goldhorn, F. (1977). Contextual influences on judgments of linear extent. Journal of Experimental Psychology: Human Perception \& Performance, 3, 105-108.

DAY, R. H. (1977). Perceptual assimilation as a basis for a class of components in geometrical illusions. In R. H. Day \& G. V. Stanley (Eds.), Studies in perception. Perth: University of West Australia Press.

Day, R. H., Bellamy, S., \& Norman, A. (1982). On the Morinaga misalignment illusion. Journal of Experimental Psychology: Human Perception \& Performance, 9, 113-125.

Pressey, A. W. (1971). An extension of assimilation theory to illusions of size, area, and direction. Perception \& Psychophysics, 9 , 172-176.

Pressey, A. W., \& Sweeney, O. (1972). Some puzzling results on the Poggendorff illusion. Perception \& Psychophysics, 12, 433-437.

RESTLE, F. (1976). Morinaga's paradox and figure-ground organization. Perception \& Psychophysics, 20, 153-156.

RoBINSON, J. O. (1972). The psychology of visual illusion. London: Hutchinson.

\section{NOTES}

1. When spatial separation increases, the area of overlap increases but the angle formed by the overlap decreases. We assume that Day et al. are referring to the angle of overlap rather than the area of overlap.

2. As one anonymous reviewer noted, subjects in our study made a number of judgments of displays with both values of spatial separation. It is conceivable that judgments of displays with one value of spatial separation affected judgments of displays with the other value. Therefore, a between-subjects design may be required to ascertain the effects of spatial separation.

(Manuscript received September 13, 1984; revision accepted for publication June 26,1985 .) 